\title{
Uniform Infinite Planar Triangulations
}

\author{
Omer Angel ${ }^{1}$, Oded Schramm ${ }^{2}$ \\ 1 Department of Mathematics, Weizmann Institute of science, Rehovot, 76100, Israel. \\ E-mail: omer@math.weizmann.ac.il \\ 2 Microsoft Corporation, One Microsoft Way, Redmond, WA 98052, USA. \\ E-mail:schramm@microsoft.com
}

Received: 13 August 2002 / Accepted: 28 February 2003

Published online: 19 September 2003 - (C) Springer-Verlag 2003

\begin{abstract}
The existence of the weak limit as $n \rightarrow \infty$ of the uniform measure on rooted triangulations of the sphere with $n$ vertices is proved. Some properties of the limit are studied. In particular, the limit is a probability measure on random triangulations of the plane.
\end{abstract}

\section{Introduction}

1.1. Motivation. What is a generic planar geometry?

There are many different planar geometries. The most commonly used one is the Euclidean plane, but is it generic? Is it more natural than, say, the hyperbolic plane?

For simplicity, consider discrete planar geometries (realized as planar graphs). Now there are still many choices. The lattice $\mathbb{Z}^{2}$ is the graph most commonly associated with planar geometry, but there is no a priori reason to prefer it over the triangular lattice, or any other lattice. One possible approach is based on convenience, preferring at each time the most convenient framework to work with. Even by that criterion no single geometry is always the best. Thus, some recent results are naturally adapted to the triangular lattice [31].

When we use a lattice, we force much more structure into our geometry than the topological condition of planarity necessitates. Random planar graphs, such as Delauny triangulations, have less enforced structure, but they still arise from the underlying Euclidean geometry. Is there a clear reason to prefer the Euclidean over the hyperbolic plane?

The approach used here is to consider a probability measure - in some sense a uniform measure - on planar geometries. Then we can ask what properties does a typical sample of that measure have. The way this is done is by considering discrete geometries, realized in the form of infinite planar triangulations, and finding an interesting distribution on them. Over finite planar triangulations the uniform measure is a natural choice. We prove the existence of a probability measure on infinite planar triangulations which is the limit 
of the uniform distributions on finite planar triangulations as their size tends to infinity. A sample of this measure is called the uniform infinite planar triangulation (UIPT). This model was suggested in [9], where Benjamini and Schramm show a.s. parabolicity of a wide class of distributions on infinite planar graphs under the condition of a uniform bound on the vertex degrees. Alas, the results there require the vertex degrees to be bounded, and hence do not apply to the UIPT.

The uniform finite planar triangulation and related objects have been studied by both combinatorists and physicists. Mathematical study is traced back to the 1960's with Tutte's attempts at the four color problem. In a series of papers Tutte was able to count the number of planar maps of a given size of various classes, including triangulations [32-35]. One of the conjectures he raised is that almost all planar maps are asymmetric, i.e., have no non-trivial automorphisms.

Tutte later proved his conjecture for a specific class of planar maps [36]. Random planar maps (and triangulations among them) have been studied extensively since then by others, proving Tutte's conjecture in a more general setting [29].

Previous research here focused on finite triangulations, but many of the results are about the asymptotic properties of planar maps and can be translated directly into claims about the infinite triangulations we study. Thus, there are results about the distribution of degrees in a uniformly chosen triangulation [18], the size of 3-connected components [8], and probabilistic 0-1 laws [7].

Schaeffer found a bijection between certain types of planar maps and labeled trees [30]. Chassaing and Schaeffer [13] recently used that bijection to show a connection between the asymptotic distribution of the radius of a random map and the integrated super-Brownian excursion. They deduce from this connection that the diameter of such a map of size $n$ scales as $n^{1 / 4}$. While they work with planar quadrangulations and we with triangulations, it appears that such local differences are insignificant when large scale observations such as diameter, growth, separation, etc. are concerned. This phenomena is referred to as universality.

The physicists study such triangulations under the title of 2-dimensional quantum gravity. The essential idea is to develop a quantum theory of gravity by extending to higher dimensions the concept of Feynman integrals on paths. Triangulations are used as a discretized version of a 2 dimensional manifold, and a function is averaged over all of them [3, 10]. More often physicists are interested not in the discretized planar triangulation but in a continuous scaling limit of it which is believed to exist.

Physicists introduced here the methods of random matrix models [15]. Through these methods and other heuristics many conjectures were made on the structure of such triangulations. In particular, it is believed that the Hausdorff dimension of the scaling limit of 2-dimensional quantum gravity is 4 [3]. For a good general exposition of quantum gravity see [2], as well as [1, 14].

Of particular interest is the KPZ relation [22] which relates critical exponents for a number of models on the plane and in 2 dimensional quantum gravity. This relation has been used to predict various exponents such as non intersection exponents for Brownian motion in the plane $[16,17]$. Later a rigorous derivation of the same values was found using the $S L E$ process [24-26].

Section 2 summarizes some results on counting triangulations which are the basis for much of what follows. Section 3 describes some properties of the UIPT that follow directly from the formulas for counting triangulations. In particular, it is shown that a.s. the UIPT has one end, i.e., the limiting process does not add any topological complications to the triangulation. 
In Sect. 4 the existence of the limit distribution is proved. In Sect. 5 we give another characterization of the UIPT by a locality property. This roughly means that different regions in the triangulation are independent of one another and that each region is uniformly distributed among all triangulations of a given size (and hence the name uniform triangulation for the infinite graph). Section 6 describes a multi-type GaltonWatson tree naturally associated with a UIPT.

In Sect. 7 we show a relation between two types of infinite planar triangulations that demonstrates the universality principle. Through this relation we also get an infinite form of the main result of [8] (see also [6]).

In a forthcoming paper [4], an alternative method of constructing and sampling the UIPT is given. Using this method, it is shown there that up to polylogarithmic factors the UIPT has growth rate $r^{4}$, agreeing both with the heuristics for the Hausdorff dimension [3] and with the asymptotics for the radius of finite maps [13]. That paper also proves that the component of the boundary of the ball of radius $r$ separating it from infinity has size roughly $r^{2}$. The method also enables an analysis of site percolation on the UIPT.

We proceed now to give formal definitions of the types of triangulations we study. An exact formulation of our main results will follow.

1.2. Definitions. The notion of a triangulation is very similar to the topological notion of a simplicial complex, although since we deal with the combinatorial aspects rather then the topological ones we will use a graph theoretic approach.

The notion of a triangulation has a bit of ambiguity around it. There are several variations on the definition, and they have much in common although there are some minor differences between them. The common thread to all variations is that a triangulation is a graph embedded in the sphere $S^{2}$ so that all faces are triangles. We will work with two types of triangulations.

Definition 1.1. Consider a finite graph $G$ embedded in the sphere $S^{2}$. A face is a connected component of $S^{2} \backslash G$. The face is a triangle if its boundary meets precisely three edges of the graph. Similarly, a face is an $\mathbf{m}$-gon if it meets $m$ edges. A triangulation $T$ is such a graph $G$ together with a subset of the triangular faces of $G$.

Let the support $S(T) \subset S^{2}$ of $T$ be the union of $G$ and the triangles in $T$. Two triangulations $T, T^{\prime}$ are considered equivalent if there is a homeomorphism of $S(T)$ and $S\left(T^{\prime}\right)$ that corresponds $T$ and $T^{\prime} . T$ is a triangulation of the sphere if $S(T)=S^{2}$. It is a triangulation of an $m$-gon if $S^{2} \backslash S(T)$ is a single $m$-gon.

For convenience, we usually abbreviate "equivalence class of triangulations" to "triangulation". This should not cause much confusion. The definition extends naturally to other manifolds, though we will not be concerned with that generality here.

Following the terminology found in [2] for types of triangulations, we define three classes of triangulations, types I, II and III. These differ according to which graphs are permitted in 1.1. In type I, there may be more than one edge connecting a pair of vertices, and loops (i.e., edges with both endpoints attached to the same vertex) are allowed as well. Type I triangulations will not be considered here, though some of the results (and proofs) apply to them as well.

Definition 1.2. A type II triangulation is a triangulation where the underlying graph has no loops, but may have multiple edges.

Definition 1.3. A type III triangulation is a triangulation where the underlying graph is a simple graph (having no multiple edges or loops). 


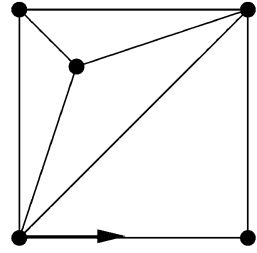

(a)

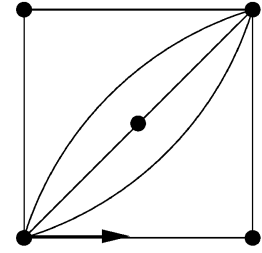

(b)

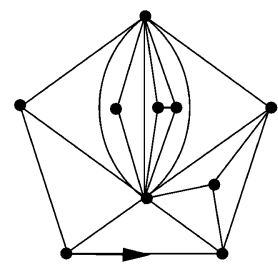

(c)

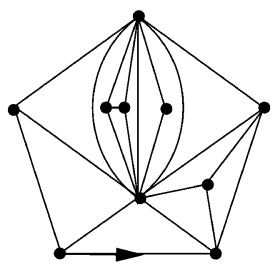

(d)

Fig. 1.1a-d. A type III and three type II triangulations. Triangulations (a) and (b) are triangulations of a square, while (c) and (d) are triangulations of a pentagon

Type II (resp. type III) triangulations are also referred to as 2-connected (resp. 3-connected) triangulations, since they are the triangulations with 2 or 3 connected underlying graphs.

If $T$ is a triangulation of a domain in the plane which may have several holes (i.e., several boundary components), we will refer to the holes of the domain as external or outer faces of $T$. An external face may have 3 vertices on its boundary and then it is a triangle in itself. In that case that face is still distinguished from the triangles of $T$. In the case of type II, an external face can also have only 2 vertices on its boundary.

It is worthwhile noting that the circle packing theorem [23] gives a canonical embedding in the sphere (up to Moebius transformations) of a type III triangulation of the sphere.

The vertices of $T$ lying on the boundary of its support $S(T)$ are called boundary vertices, and those in the interior of $S(T)$ are internal vertices. When we consider triangulations of a domain in the sphere with a number of boundary components we will usually fix the number of boundary vertices in each component as part of the domain. Thus, for example, a disc with $m$ boundary vertices will be distinguished from a disc with $m^{\prime} \neq m$ boundary vertices. Such a disc is referred to as an $m$-gon.

The size of a triangulation $T$, denoted $|T|$, is defined as the number of internal vertices. Since all faces are triangles, by Euler's characteristic formula, if $E$ (resp. $F$ ) is the number of edges (resp. faces) of $T$, then $3|T|-E$ (resp. $2|T|-F$ ) is determined by the number and size of the boundary components of $|T|$. In particular, for a sphere all vertices are internal, and so $3|T|-E=6$ and $2|T|-F=4$.

Note that for a type III triangulation of the sphere (and even slightly more generally) the underlying graph determines the triangulation, i.e., whether any three edges form a triangle or not. When multiple edges are allowed there may be several distinct embeddings of the graph in the sphere giving distinct triangulations. E.g., in Fig. $1.1 \mathrm{c}$ and $\mathrm{d}$ are distinct triangulations that have the same underlying graph.

A fundamental problem encountered when studying planar maps (triangulations included) is that of symmetries, namely that some maps have non-trivial automorphism groups. It seems plausible that most triangulations are asymmetric. While this has been proved [36, 29], we dispose of this problem in another manner. A simple way of eliminating any symmetries there is by adding a root to the triangulation.

Definition 1.4. A root in a triangulation $T$ consists of a triangle $t$ of $T$ called the root face, with an ordering of its vertices $(x, y, z)$. The vertex $x$ is the root vertex and the directed edge $(x, y)$ is the root edge.

Note that in type II triangulations there may be more than one triangle with the same three vertices, so marking only the three vertices does not generally suffice. In 
a triangulation of the sphere, if the root edge is given, then there are exactly two possibilities for the root. We will usually mark only the root edge as in Fig. 1.1, by an arrow.

When $T$ has a boundary we will usually assume that the root edge lies on the boundary. Since a disc with $m$ boundary vertices is referred to as an $m$-gon, triangulations (a) and (b) of Fig. 1.1 are of a square, while (c) and (d) are of a pentagon.

There are many possible variations on the definition of triangulation. Restricting to 2 or 3-connected underlying graphs (or even 4 or 5-connected) gives slightly different definitions. It is possible to restrict the degrees of vertices, or to allow faces that are not triangles. General convex polytopes can thus be described as a variation on the notion of triangulation. Most of the results proved below should have analogues for such generalizations, though the proofs do not always carry through. For convenience and brevity, we will deal with type II and type III triangulations here.

The definition of rooted infinite triangulations is very similar to that of finite triangulations. In that case $G$ is infinite, of course, but we always require it to be locally-finite; that is, each vertex is incident to only finitely many edges. The only slightly technical point is that we require the embedding of $G$ to be faithful to the combinatorial structure, in the following sense: if $\left\{p_{n}: n=1,2, \ldots\right\}$ is a sequence in $S^{2}$ belonging to distinct edges of $G$, then accumulation points of $p_{n}$ must be outside of $S(T)$.

A triangulation may be endowed with a metric in a number of ways. We will rather use a metric on the vertices of a triangulation - the graph metric induced by the underlying graph. It is also interesting to consider a triangulation as a metric space by having each face be isometric to an equilateral triangle with the shortest path metric on the whole triangulation. Then a triangulation of the sphere is a metric space homeomorphic to the sphere.

For either type, the space of finite and infinite (equivalence classes of) connected planar rooted triangulations is endowed with a natural topology described in [9]. (Note however, that there the root was only a vertex, leading to a closely related but slightly different distribution). A sequence of rooted triangulations converges to a triangulation $T$ if eventually they are equivalent with $T$ on arbitrarily large combinatorial balls around the root. This is a metric topology: e.g., set $d\left(T, T^{\prime}\right)=k^{-1}$, where $k$ is the maximal radius such that the combinatorial balls of radius $k$ around the roots are equivalent. In this topology, all finite triangulations are isolated points, and infinite triangulations are their accumulation points. We leave it to the reader to verify that limits of triangulations of the sphere are triangulations of domains in the plane.

Unlike in the setup of [9], the triangulation space is not compact. Consider the sequence $T_{n}$ of triangulations where $T_{n}$ contains two vertices of degree $n$ with the same $n$ neighbors forming a cycle (i.e., a double pyramid). Since $T_{n}$ are distinct and all have diameter $2,\left\{T_{N}\right\}$ has no convergent subsequence.

We will be interested in the uniform distributions on triangulations:

Definition 1.5. $\tau_{n}^{2}$ (resp. $\left.\tau_{n}^{3}\right)$ is the uniform distribution on rooted type II (resp. type III) triangulations of the sphere of size $n$ (i.e., having $n$ vertices).

The topology on the triangulation space induces a weak topology on the linear space of measures supported on planar triangulations. We study the distribution on infinite planar triangulations which is the weak limit of $\tau_{n}$ as $n \rightarrow \infty$. The weak limit may equivalently be defined as a limit with respect to neighborhoods of the root, i.e.,

Definition 1.6. A measure supported on rooted triangulations $\tau$ is a weak limit of a sequence of measures $\tau_{n}$ if for any radius $r$ and any finite triangulation $T$ : 


$$
\lim _{n \rightarrow \infty} \tau_{n}\left(B_{r}(0)=T\right)=\tau\left(B_{r}(0)=T\right),
$$

where $B_{r}(0)$ is the ball of radius $r$ around the root vertex (0) in the graph metric.

This definition is equivalent to the test-function definition that for every continuous function $f$ on the space of triangulations:

$$
\int f d \tau_{n} \rightarrow \int f d \tau
$$

If $T_{1}$ and $T_{2}$ are rooted triangulations, we say that $T_{1}$ is contained in $T_{2}$ (and write $T_{1} \subset T_{2}$ ) if the two roots are the same and $T_{1}$ is contained in $T_{2}$ as unrooted triangulations. Sometimes we may also write $T_{1} \subset T_{2}$ to mean that there is a triangulation isomorphic to $T_{1}$ contained in $T_{2}$.

Finally, a word on notation. By $X_{n} \sim Y_{n}$ we mean that $X_{n} / Y_{n} \rightarrow 1$. By $X_{n} \approx Y_{n}$ we mean that $\log X_{n} / \log Y_{n} \rightarrow 1$. We use $c, c_{1}, c_{2}, \ldots$ to signify constants, whose actual value may change from one formula to another.

\subsection{Main Results. We will first prove that}

Theorem 1.7. There exists a probability measure $\tau^{2}$ (resp. $\tau^{3}$ ) supported on infinite planar triangulations of type II (resp. type III) such that

$$
\tau^{i}=\lim \tau_{n}^{i}
$$

Note. If $\tau_{n}$ are distributions on rooted graphs with uniformly bounded degrees, then since there are finitely many possibilities for $B_{r}(0)$, by compactness $\tau_{n}$ has a subsequential limit. In our case the triangulations do not have uniformly bounded degrees. We prove a limit exists - not just a subsequential limit. Additionally we prove that the limit is a probability measure; this is not a priori clear because of the lack of compactness.

Having defined the limit measure $\tau$ (we will often drop the type notation when results hold for either type) we turn to study the a.s. properties of a sample of $\tau$. Denote such a sample by UIPT. A basic geometric property, one endedness, will show that the limit structure maintains the plane's topology. Recall the definition:

Definition 1.8. A graph $G$ is said to have one end (is one-ended) if for any finite subgraph $H, G \backslash H$ contains exactly one infinite connected component.

Theorem 1.9. The UIPT is a.s. one ended, and is therefore a triangulation of the plane.

We also ask about the electrical type of the underlying graph. In [9] it is shown that for any sequence of distributions on planar graphs with degrees uniformly bounded by $M$, if a root is marked uniformly in each graph then every subsequential limit is a.s. recurrent. This holds, for example, for planar triangulations with uniformly bounded degrees. However, for those distributions it is not clear how to prove that the limit exists (simulations support this [11]). The following conjectures appear in [9]:

Conjecture 1.10. For every $M \geq 6$, the distributions $\tau_{N}$ conditioned to have degrees uniformly bounded by $M$ are weakly convergent. 


\section{Conjecture 1.11. The UIPT is a.s. recurrent.}

VEL parabolicity (for vertex extremal length) is a property of infinite graphs, closely related to circle packings for planar graphs. In graphs with bounded degrees it is equivalent to recurrence [21]. The proof in [9] of a.s. VEL parabolicity for uniform infinite triangulations with bounded degrees is still valid for the UIPT, with tightness (Lemma 4.4) filling the role of bounded degrees.

\section{Counting}

2.1. Classical results. Much of the analysis of triangulations is based on counting them. This is true both for finite triangulations and for infinite triangulations where the asymptotics of the finite triangulations come into play. The following counting results go back to Tutte [32] who counted various types of planar maps and triangulations. The results we use here are not due to Tutte but are derived using the same technique he uses. More details can be found in [12]. A good account of the technique including all results given here can be found in [20].

Theorem 2.1. 1. For $n, m \geq 0$, not both 0 , the number of type II triangulations of a disc with $m+2$ boundary vertices and $n$ internal vertices that are rooted on a boundary edge is

$$
\varphi_{n, m}^{2}=\frac{2^{n+1}(2 m+1) !(2 m+3 n) !}{m !^{2} n !(2 m+2 n+2) !} .
$$

2. For $m \geq 1, n \geq 0$, the number of rooted type III triangulations of a disc with $m+2$ boundary vertices and $n$ internal vertices that are rooted on a boundary edge is

$$
\varphi_{n, m}^{3}=\frac{2(2 m+1) !(4 n+2 m-1) !}{(m-1) !(m+1) ! n !(3 n+2 m+1) !} .
$$

The case $n=m=0$ for type II triangulations warrants special attention. A triangulation of a 2-gon must have at least one internal vertex so there are no triangulations with $n=m=0$, yet the above formula gives $\varphi_{0,0}^{2}=1$. It will be convenient to use this value rather than 0 for the following reason. Typically, a triangulation of an $m$-gon is used not in itself but is used to close an external face of size $m$ of some other triangulation by "gluing" the boundaries together. When the external face is a 2-gon, there is a further possibility of closing the hole by gluing the two edges to each other with no additional vertices. Setting $\varphi_{0,0}^{2}=1$ takes this possibility into account.

Since we will consider the asymptotics of large triangulations we will need the following estimates of these numbers. Using the Stirling formula, as $n \rightarrow \infty$ we have the following:

$$
\varphi_{n, m}^{2} \sim C_{m}^{2} \alpha_{2}^{n} n^{-5 / 2}
$$

where $\alpha_{2}=27 / 2$ and

$$
C_{m}^{2}=\frac{\sqrt{3}(2 m+1) !}{4 \sqrt{\pi} m !^{2}}(9 / 4)^{m} \sim C 9^{m} m^{1 / 2} .
$$

For type III triangulations we have similar estimates:

$$
\varphi_{n, m}^{3} \sim C_{m}^{3} \alpha_{3}^{n} n^{-5 / 2},
$$


where $\alpha_{3}=256 / 27$ and as $m \rightarrow \infty$ :

$$
C_{m}^{3}=\frac{2(2 m+1) !}{6 \sqrt{6 \pi}(m-1) !(m+1) !}(16 / 9)^{m} \sim C(64 / 9)^{m} m^{1 / 2} .
$$

Much of the time we will not distinguish between type II and type III triangulations. The type index will be dropped either when the stated results hold for both types or when it is clear which type is discussed.

We are interested in triangulations of the sphere that have no predefined boundary. The number of those is given by:

Proposition 2.2. For either type, the number of rooted triangulations of the sphere with $n$ vertices is $\varphi_{n-3,1}$.

Proof. Adding a triangle that closes the outer face of a triangulation of a triangle makes a triangulation of the sphere. Alternatively, removing the triangle incident on the root edge that is not the root triangle gives a triangulation of a triangle rooted on the boundary. Thus, there is a bijection between triangulations of the sphere with $n$ vertices and triangulations of a triangle with $n-3$ internal vertices.

We will also be interested in triangulations of discs where the number of internal vertices is not prescribed. The following measure is of particular interest:

Definition 2.3. The free distribution on rooted triangulations of an $(m+2)$-gon, denoted $\mu_{m}$, is the probability measure that assigns weight

$$
\alpha^{-n} / Z_{m}\left(\alpha^{-1}\right)
$$

to each rooted triangulation of the $(m+2)$-gon having $n$ internal vertices, where

$$
Z_{m}(t)=\sum_{n} \varphi_{n, m} t^{n}
$$

As before, $\mu_{m}^{2}$ (resp. $\mu_{m}^{3}$ ) will denote free type II (resp. type III) triangulations, and similarly for the partition functions $Z_{m}^{2}$ and $Z_{m}^{3}$. Thus, the probability of a triangulation $T$, is proportional to $\alpha^{-|T|}$, and $Z_{m}$ acts as a normalizing factor.

Note that by the asymptotics of $\varphi$ as $n \rightarrow \infty$ we see that the sum defining $Z$ converges for any $t \leq \alpha^{-1}$ and for no larger $t$. The value of the partition functions will be useful. For this we have:

Proposition 2.4. 1. For type II triangulations, if $t=\theta(1-2 \theta)^{2}$ :

$$
Z_{m}^{2}(t)=\frac{(2 m) !((1-6 \theta) m+2-6 \theta)}{m !(m+2) !}(1-2 \theta)^{-(2 m+2)} .
$$

2. For type III triangulations, if $t=\theta(1-\theta)^{3}$ :

$$
Z_{m}^{3}(t)=\frac{(2 m) !((1-4 \theta) m+6 \theta)}{m !(m+2) !}(1-\theta)^{-(2 m+1)} .
$$


At the critical point $t=\alpha^{-1}$ we will omit $t$. There $Z$ takes the values:

$$
Z_{m}^{2}=Z_{m}^{2}(2 / 27)=\frac{(2 m) !}{m !(m+2) !}\left(\frac{9}{4}\right)^{m+1}
$$

and

$$
Z_{m}^{3}=Z_{m}^{3}(27 / 256)=\frac{2(2 m) !}{m !(m+2) !}\left(\frac{16}{9}\right)^{m}
$$

The proof can be found as intermediate steps in the derivation of $\varphi_{m, n}$ in [20]. The above form may be deduced after a suitable reparametrization of the form given there.

2.2. Universality. While the exponential term in the asymptotics of $\varphi$ is different for type II and III, the next term of $n^{-5 / 2}$ is the same. Similarities also occur in the asymptotics of $C_{m}$ and of $Z_{m}$ for the two types. Those similarities are not coincidental. It turns out that the asymptotic form is quite common when counting 2 dimensional structures. That form of the asymptotics is not dependent on the manifold, and is valid for any 2-dimensional manifold with or without boundaries. The same forms also appear when instead of triangulations other types of maps are considered, and was found to hold for a large variety of map types $([11,10]$ and also the result of [13], related to our growth results). We therefore believe that many of the results here hold in a much more general context.

This universality is related to the basic property of the 2-sphere that a cycle partitions it into two parts, i.e., the Jordan Curve Theorem. This leads to a similarity between recurrence relations for different types of structures and through them to similar asymptotics for the solutions. For another instance of universality and some explanation see $[5,6]$.

It turns out that the exponential part of the asymptotics will cancel out often and when finer properties of infinite triangulations are considered the power term will come into play and determine the observed behavior.

2.3. Some estimates. We will need the following estimates throughout the paper.

Lemma 2.5. Let

$$
S(k, N, a)=\sum_{\substack{n_{1}+\ldots+n_{k}=N \\ n_{1}, n_{2}>a}}\left(\prod n_{i}\right)^{-5 / 2}
$$

then for any $k$ there is a $c=c(k)$ such that for any $N$ :

$$
S(k, N, a) \leq c N^{-5 / 2} a^{-3 / 2} \text {. }
$$

Proof. Clearly

$$
S(k, N, a) \leq k ! \sum_{\substack{n_{1} \geq \ldots \geq n_{k} \\ n_{1}+\ldots+n_{k}=N \\ n_{1}, n_{2}>a}}\left(\prod n_{i}\right)^{-5 / 2}
$$

since each term in the sum over ordered $k$-tuples corresponds to at most $k$ ! terms in the original sum, and less if there are any repetitions. Since each possible choice of 
$n_{2}, \ldots, n_{k}$ determines a unique value for $n_{1}$ and always $n_{1} \geq N / k$ we can replace $n_{1}$ by the smaller $N / k$ and extend the range of summation.

$$
\begin{aligned}
S(k, N, a) & \leq k !(N / k)^{-5 / 2} \sum_{\substack{n_{2} \geq \ldots \geq n_{k} \\
n_{2}>a}}\left(\prod_{i \neq 1} n_{i}\right)^{-5 / 2} \\
& \leq k !(N / k)^{-5 / 2}\left(\sum_{n_{2} \geq a} n_{2}^{-5 / 2}\right) \prod_{i>2}\left(\sum_{n_{i}} n_{i}^{-5 / 2}\right) \\
& \leq c N^{-5 / 2} a^{-3 / 2} .
\end{aligned}
$$

\section{Basic Properties}

3.1. Invariance with respect to the random walk. If we are given a finite triangulation, but not the location of the root, what can we say about the location of the root? The following proposition says: not much. For a triangulation $T$ and a possible root $r$ in $T$ let $T_{r}$ denote the triangulation $T$ with $r$ marked as root (if $T$ is rooted then the old root is no longer marked).

Proposition 3.1. Let $T$ be a sphere triangulation chosen by $\tau_{n}$, and $r$ be a root in $T$ chosen uniformly among all possible roots. Then $T_{r}$ is uniformly distributed among all rooted triangulations (of size $n$ ).

Proof. At first glance this seems trivial: since all rooted triangulations are equally likely no triangle in $T$ should be more likely to be the root than any other. However, there is a subtlety here since there may be several triangles $r$ such that the triangulations $T_{r}$ are isomorphic. This occurs whenever $T$ has a non trivial automorphism.

The key fact here is that any automorphism of $T$ that preserves a root is necessarily the identity automorphism. If $R$ is the set of possible roots and $G$ is the automorphism group of $T$ as an unrooted triangulation, then $G$ acts naturally on $R$ and a non identity element of $G$ has no fixed points in $R$. Thus, the size of the orbit of a triangle $r \in R$ is just the size of $G$, regardless of $r$.

Since each of the orbits in the action of $G$ on $R$ corresponds to a distinct rooted triangulation, and each orbit has the same size, each possible triangulation is equally likely to result after a new root is selected.

Note that since each directed edge can be completed in two ways to a root each directed edge is equally likely to be the root edge. From this we see that the UIPT must be invariant with respect to a random walk:

Theorem 3.2. Let $T$ be a triangulation chosen by $\tau_{N}$ for some $N$ or by a subsequential limit $\tau$. If $x$ is the root vertex of $T, y$ is a uniformly chosen neighbor of $x$, and $(y, z, w)$ is a triangle in $T$ uniformly chosen among all triangles including $y$, then $T_{(y, z, w)}$ has the same law as $T$.

Proof. For finite $N$, if a vertex $x$ of degree $d$ is the root vertex, then there are $d$ possibilities for the root edge (and $2 d$ options for the root). It follows that the probability that $x$ is the root is proportional to its degree. This is the stable distribution for the random 
walk on the graph of $T$, so as a consequence of Proposition 3.1 we see that $T_{(y, z, w)}$ has the same law as $T$.

Since this is true for every $\tau_{N}$, the same holds for any subsequential limit.

3.2. One endedness. We start with a lemma describing the behavior of a triangulation on a disjoint union of discs.

Lemma 3.3. Given $k$ disjoint polygons (with given boundary sizes) and a triangulation $T$ of the polygons, let $n_{i}$ be the number of internal vertices in the $i^{\text {th }}$ polygon. Then

$$
\left|\left\{T \mid \sum n_{i}=N \wedge \exists i, j, i \neq j, n_{i}, n_{j}>a\right\}\right|<C \alpha^{N} N^{-5 / 2} a^{-3 / 2},
$$

where $C$ depends only on the number and sizes of the boundaries of the polygons.

Proof. We prove that the number of triangulations where $n_{1}, n_{2}>a$ is small, as required. By symmetry, the number for any other pair $(i, j)$ has the same bound. Since the number of such pairs, $\left(\begin{array}{l}k \\ 2\end{array}\right)$, does not depend on $a$ or on $N$, this suffices.

We use the upper bound $\varphi_{n, m} \leq \beta_{m}(n+1)^{-5 / 2} \alpha^{n}(+1$ is only necessary to account for $n=0$, and is not essential). Assume the $i^{\text {th }}$ domain has boundary size $m_{i}+2$. The number of triangulations we wish to bound is:

$$
\begin{aligned}
\sum_{\substack{n_{1}+\ldots+n_{k}=N \\
n_{1}, n_{2}>a}} \prod_{i} \varphi_{n_{i}, m_{i}} & \leq \sum_{\substack{n_{1}+\ldots+n_{k}=N \\
n_{1}, n_{2}>a}} \prod_{i} \beta_{m_{i}}\left(n_{i}+1\right)^{-5 / 2} \alpha^{n_{i}} \\
& =\alpha^{N} \prod_{\substack{m_{i} \\
n_{1}+\ldots+n_{k}=N \\
n_{1}, n_{2}>a}} \prod\left(n_{i}+1\right)^{-5 / 2} \\
& \leq c_{1} \alpha^{N} \sum_{\substack{n_{1}+\ldots+n_{k}=N+k \\
n_{1}, n_{2}>a}} \prod\left(n_{i}\right)^{-5 / 2} \\
& \leq c_{2} \alpha^{N} N^{-5 / 2} a^{-3 / 2}
\end{aligned}
$$

where at the end we used Lemma 2.5.

Generally a limit $T$ of a sequence of finite sphere triangulations need not have support $S(T)$ which is homeomorphic to the sphere or even the plane. While the limit is still planar, when embedded in the sphere $S(T)$ may have any number of accumulation points. One accumulation point gives a punctured sphere, i.e., the plane. More than one means that $S(T)$ has a more complicated topological structure; it is no longer simply-connected.

Corollary 3.4. Every subsequential limit of $\tau_{N}$ a.s. has one end.

Proof. Suppose that a subsequential limit $\tau$ has more than one end with positive probability. Then for some $k$ and some $\varepsilon>0$ the probability that a loop of length $k$ including the root partitions a sample of $\tau$ into two infinite parts is at least $\varepsilon$. This implies that for any $a$ for infinitely many $N$ the $\tau_{N}$-probability of having a loop of length $k$ including the root that has at least $a$ vertices on either side is at least $\varepsilon / 2$. Call such a loop a separating loop.

Count pairs $(T, L)$ with $T$ a triangulation of size $N$ and $L$ a separating loop included in $T$. From Lemma 3.3 we know that the total number of such pairs is $O\left(\alpha^{N} N^{-5 / 2} a^{-3 / 2}\right)$. However, the number of sphere triangulations with $N$ vertices is $\varphi_{N-3,1} \sim C \alpha^{N} N^{-5 / 2}$, and by dividing we deduce that the expected number of separating loops is $O\left(a^{-3 / 2}\right)$. In particular as $a \rightarrow \infty$ the probability that a separating loop exists tends to 0 . 


\section{Existence of the Limit}

4.1. Tightness. The difficulty in establishing the existence of the limit distribution as a probability measure is showing that the size of the ball of radius $r$ around the root is tight with respect to the total number of vertices $N$. Recall that a family of random variables $X_{n}$ is tight with respect to $n$ if

$$
\lim _{t \rightarrow \infty} \mathbb{P}\left(\left|X_{n}\right|>t\right)=0
$$

uniformly with respect to $n$.

To this end, we first prove the following estimates for the degree of the root in either type of triangulation. While the lemmas are very similar in nature, the methods of proof given here are different. This demonstrates the underlying unity of the different models, while local differences make some techniques applicable in one and others in another.

The following lemmas appear in similar form in [18].

Lemma 4.1. Denote the degree of the root vertex by $d_{0}$. For any $\varepsilon>0$ there is a $c=c(\varepsilon)$ such that

$$
\tau_{N}^{3}\left(d_{0}=k\right)<c\left(\frac{3}{4}+\varepsilon\right)^{k}
$$

uniformly for all $N$ and

$$
\tau_{N}^{3}\left(d_{0}=k\right) \underset{N \rightarrow \infty}{\stackrel{\text { unif }}{\longrightarrow}} \frac{2(2 k-3) !}{(k-3) !(k-1) !}\left(\frac{3}{16}\right)^{k-1} .
$$

Proof. A type III triangulation of the sphere where the root vertex has degree $k$ is the union of two triangulations, $T_{0}, T_{1}$ whose intersection is a $k$-gon: $T_{0}$ contains the root vertex and $k$ triangles connecting it to the sides of the $k$-gon, and $T_{1}$ contains all other triangles. The root triangle has one edge in the intersection of $T_{0}$ and $T_{1}$. Choose this edge to be the root edge of $T_{1}$. Now $T \leftrightarrow T_{1}$ is a bijection between rooted triangulations of the sphere with $d_{0}=k$ and rooted triangulations of a $k$-gon with the root edge on the boundary.

If $|T|=N$, then $\left|T_{1}\right|=N-k-1$, and we know the number of such triangulations. Dividing by the number of sphere triangulations, we get:

$$
\begin{aligned}
\tau_{N}\left(d_{0}=k\right) & =\frac{\varphi_{N-1-k, k-2}}{\varphi_{N-3,1}} \\
& =\frac{2(2 k-3) !}{3(k-3) !(k-1) !} \frac{(4 N-2 k-9) !(3 N-6) !(N-3) !}{(4 N-11) !(3 N-k-6) !(N-k-1) !} \\
& \rightarrow \frac{2(2 k-3) !}{(k-3) !(k-1) !}\left(\frac{3}{16}\right)^{k-1} .
\end{aligned}
$$

To prove the uniform exponential bound consider the ratio

$$
\frac{\tau_{N}\left(d_{0}=k+1\right)}{\tau_{N}\left(d_{0}=k\right)}=\frac{(2 k-1)(2 k-2)(3 N-k-6)(N-k-1)}{k(k-2)(4 N-2 k-9)(4 N-2 k-10)}<\frac{3}{4}+\varepsilon
$$

for any sufficiently large $N, k$. 


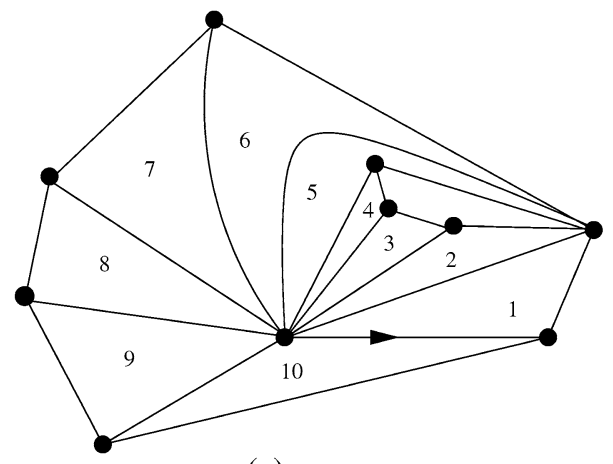

(a)

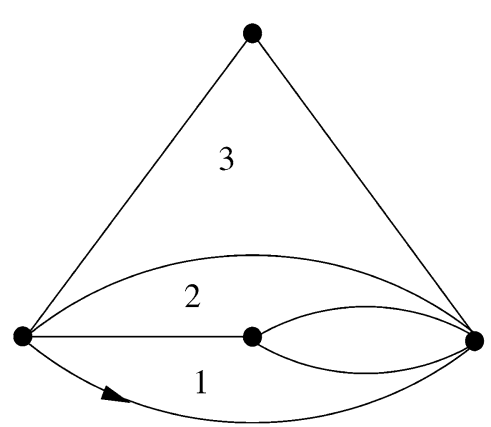

(b)

Fig. 4.1a, b. Proof of the tightness of the root's degree

For type II, since multiple edges are present, there are two notions of degree. The vertex degree of $v$ is the number of neighbors it has, while the edge degree of $v$ is the number of edges incident on it. For our purposes bounding the vertex degree is sufficient, but in what follows we bound the larger edge degree.

Lemma 4.2. Denote the edge degree of the root vertex by $d_{0}$, then there is a c such that

$$
\tau_{N}^{2}\left(d_{0}=k\right)<c\left(\frac{5}{3 \sqrt{3}}\right)^{k}
$$

uniformly for all $N$.

Proof. Let $t_{1}, \ldots, t_{d_{0}}$ be the triangles incident with the root vertex, ordered counterclockwise starting with the root triangle $t_{1}$. For $s \leq d_{0}$ let $T_{s}$ be the sub-triangulation including triangles $t_{1}, \ldots, t_{s}$. Adding $t_{i}$ one at a time, we consider the distribution of $T_{s+1}$ conditioned on $T_{s}$, and show that for any $T_{s}$ there is a probability bounded away from 0 that $d_{0}=s+1$.

$T_{S}$ may have several external faces. One of those, say $F$, includes the root vertex, and $t_{s+1}$ is in $F$. In order for $t_{s+1}$ to be the last triangle adjacent to the root vertex it must include the two edges of $F$ on either side of the root vertex. Thus, in Fig. 4.1a, the triangles incident with the root vertex are numbered. The triangle $t_{10}$ is the final triangle, and it includes both the edge from $t_{9}$ and the edge from the root triangle $t_{1}$. Note that when triangle $t_{5}$ is added, an unknown part of the triangulation is enclosed, but this will not effect the bounds we get.

To bound from below the probability that $t_{s+1}$ is the last triangle conditioned on $T_{s}$, assume that the boundary of $F$ has size $m+2$. A first condition on the events that the part of the triangulation inside $F$ has $n$ vertices. The number of possible ways to triangulate $F$ under these constraints is $\varphi_{n, m}$. If $t_{s+1}$ is the last triangle around the root, then adding it leaves a face of boundary size $m+1$ with $n$ internal vertices. Thus the probability of the next triangle being the last one is:

$$
\begin{aligned}
\frac{\varphi_{n, m-1}}{\varphi_{n, m}} & =\frac{m^{2}(2 n+2 m+1)(2 n+2 m+2)}{2 m(2 m+1)(3 n+2 m-1)(3 n+2 m)} \\
& >\frac{2 m(n+m)^{2}}{(2 m+1)(3 n+2 m)^{2}} .
\end{aligned}
$$


If $m>0$, then this is at least $2 / 27$, so the probability that $d_{0}>s+1$ is at most $25 / 27$. Since this bound is uniform it also holds when conditioning only on $T_{S}$ and not on the number of internal vertices in $F$.

Thus, as new triangles are revealed, each triangle has a probability of at least 25/27 of being the last one, unless $m=0$. If $m=0$, as for $T_{2}$ in Fig. $4.1 \mathrm{~b}$, then after a triangle is added we must have $m=1$ and so out of every two consecutive $s$, at least one has $m>0$. It follows that the probability of having more than $k$ edges leaving the root vertex is at most $(25 / 27)^{(k-1) / 2}$, as claimed.

Note. For type III triangulations, Lemma 4.1 gives the exact probability of any given degree in the UIPT. To a large extent, this is possible because the radius 1 neighborhood of the root has a simple structure. When multiple edges are allowed, even the ball of radius 1 around the root can have a complicated structure, making an exact calculation harder to get. On the other hand, for type II triangulations, we can calculate the exact probability that a certain triangle is present in the triangulation conditioned on some sub-triangulation (e.g., the probability that $t_{s+1}$ is the last triangle around the root conditioned on $T_{s}$, as in the proof). This is much harder to do for type III triangulations, because we need to keep track of which pairs of vertices already have edges between them, whereas in type II triangulations adding another edge is always legal.

At this point we will rigorously define the ball $B_{r}$ of radius $r$ around the root (or any other vertex, for that matter). This ball is a sub-triangulation, but there is some subtlety in its definition. The vertices of $B_{r}$ are all those vertices at distance at most $r$ from the root vertex, but not all edges and triangles between these vertices are necessarily part of $B_{r}$.

Definition 4.3. $B_{0}$ is just the root vertex itself. $B_{r+1}$ is composed of all triangles incident on any vertex of $B_{r}$ together with their vertices and edges.

Note that there may be edges between vertices on the boundary of $B_{r}$ that are not part of $B_{r}$ itself. Next, we turn our attention to the size of the ball $B_{r}$. The following lemma holds for both types.

Lemma 4.4. For any fixed $r$ the random variables $M_{r}=\max \left\{d_{v} \mid v \in B_{r}\right\}$ (i.e., the maximal degree in $B_{r}$ ) defined on the space of triangulations with measure $\tau_{N}$ are tight with respect to $N$.

Proof. For $r=0, B_{r}$ is just the root, and Lemmas 4.1 and 4.2 show that the degree of the root is tight with respect to $N$ for either type.

We proceed by induction on $r$. Suppose that $M_{r}$ is tight with respect to $N$. To show that $M_{r+1}$ is also tight we use Theorem 3.2. Let $T$ denote a sample of $\tau_{n}$, and let $X_{0}, X_{1}, \ldots$ be a simple random walk on $T$ started at the root vertex $X_{0}$. Denote by $\mathbb{P}$ the resulting probability measure on triangulations with paths beginning at the root. It follows from Theorem 3.2 that for any $i$ the degree of $X_{i}$ has the same distribution as the degree of the root. Fixing $M^{\prime}>M>0$ we estimate the probability that $M_{r} \leq M$ and yet $M_{r+1}>M^{\prime}$. Conditioned on this event, there is at least one vertex $u \in B_{r+1} \backslash B_{r}$ with $d_{u}>M^{\prime}$. Since there is a path of length $r+1$ from the root vertex to $u$, and all vertices on the path have degrees at most $M$,

$$
\mathbb{P}\left(d_{X_{r+1}}>M^{\prime} \mid M_{r} \leq M<M^{\prime}<M_{r+1}\right) \geq M^{-(r+1)},
$$

and so

$$
\mathbb{P}\left(d_{X_{r+1}}>M^{\prime}\right) \geq M^{-(r+1)} \mathbb{P}\left(M_{r} \leq M<M^{\prime}<M_{r+1}\right) .
$$


By Theorem 3.2 the LHS does not depend on $r$ and is simply $\tau_{n}\left(d_{0}>M^{\prime}\right)$. The RHS does not depend on the random walk either, so for any $M$,

$$
M^{r+1} \tau_{n}\left(d_{0}>M^{\prime}\right) \geq \tau_{n}\left(M_{r+1}>M^{\prime}\right)-\tau_{n}\left(M_{r}>M\right) .
$$

By induction, for all $\varepsilon>0$ we may choose $M=M(\varepsilon)$ such that $\tau_{n}\left(M_{r}>M\right) \leq \varepsilon / 2$ for all $n$. Then we take $M^{\prime}=M^{\prime}(\varepsilon)>M$ sufficiently large so that $\tau_{n}\left(d_{0}>M^{\prime}\right)<$ $M^{-(r+1)} \varepsilon / 2$ for all $n$. This gives $\tau_{n}\left(M_{r+1}>M^{\prime}\right)<\varepsilon$ for all $n$, and completes the proof.

Corollary 4.5. For any fixed $r$ the random variables $\left|B_{r}\right|$ are tight with respect to $N$.

Proof. Since $M_{r}$ is tight with respect to $N$, and $\left|B_{r}\right|<\left(1+M_{r}\right)^{r},\left|B_{r}\right|$ is tight as well.

Corollary 4.6. Every subsequential limit of $\tau_{N}$ is a probability measure.

In [28] it is shown that for every finite triangulation $T$ there is a constant $c$ such that asymptotically, in almost every sphere triangulation of size $n$ the number of times $T$ appears is roughly $c n$. This $c(T)$ is roughly the probability that a neighborhood of the root in the UIPT is isomorphic to $T$. In fact, the result of [28] is stronger, since it gives not just an annealed probability of seeing $T$ but that the quenched probability is constant. We bring here a simpler calculation just for the annealed probability, since the results of the calculation are useful in what follows.

It will be easier to work with rooted triangulations having the property that if they are a sub-triangulation of the UIPT, then they appear in it exactly once. This is not always the case: a root triangle together with a cycle of some length may appear in the triangulation in several different ways.

Definition 4.7. A rooted triangulation $A$ is rigid if it is connected and no triangulation includes two distinct copies of $A$ with coinciding roots.

The balls $B_{r}$ of a triangulation are rigid, as is evident from the following sufficient criterion for rigidity (the proof is left to the reader).

Lemma 4.8. If in the dual graph of triangulation $A$ the vertices corresponding to the triangles of A form a connected set, and every vertex of $A$ is incident on a triangle, then A is rigid.

This criterion is not necessary for rigidity, as is demonstrated by Fig. 4.2 a, where there is an isolated triangle. In fact a sufficient and necessary criterion is that the support $S(T)$ be 3-connected. In order to complete a planar triangulation to a sphere triangulation we need to fill each of its external faces with some triangulation. The advantage of rigid triangulations is that filling the external faces in different ways must lead to distinct sphere triangulations, whereas for non-rigid triangulations different ways of filling the faces may give rise to the same complete triangulation. Figure $4.2 \mathrm{~b}, \mathrm{c}$ give an example of a non-rigid triangulation and how two completions give rise to the same triangulation.

A second consequence of the construction of $B_{r}$, whose proof (an application of the Jordan curve theorem) is left to the reader is:

Lemma 4.9. In the ball $B_{r}$ there are no edges between two vertices of any external face except those making the face itself. 


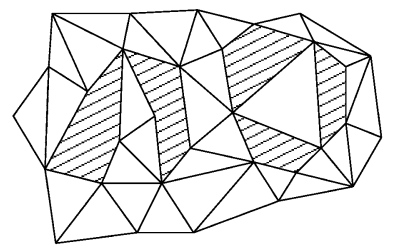

(a)

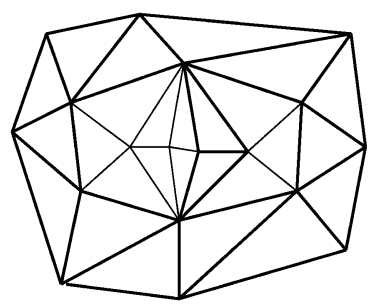

(b)

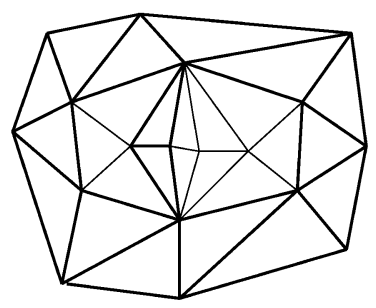

(c)

Fig. 4.2a-c. A rigid triangulation (with shaded outer faces) and two isomorphic completions of a non-rigid triangulation

Proposition 4.10. Let $A$ be a rigid rooted triangulation having no edges between two vertices of an external face except those making the face itself. Assume A has $n$ vertices, some of which are on $k$ external boundary components of sizes $m_{1}+2, \ldots, m_{k}+2$. Then every subsequential limit $\tau$ of $\tau_{N}$ has:

$$
\tau(A \subset T)=\frac{\alpha^{3-n}}{C_{1}}\left(\prod Z_{m_{i}}\right) \sum \frac{C_{m_{i}}}{Z_{m_{i}}} .
$$

Moreover, the probability that the $i^{\text {th }}$ face is the infinite one corresponds to the $i^{\text {th }}$ term in the sum, i.e.:

$$
\frac{\alpha^{3-n}}{C_{1}} C_{m_{i}} \prod_{j \neq i} Z_{m_{j}}
$$

Note. For type II triangulations the restriction on edges between vertices of an external face is not necessary. For type III triangulations it is needed, since when such an edge exists it imposes restrictions on the component inside the face. In general, the probability that $A \subset T$ can be found using the proposition together with the inclusion-exclusion principle. The requested probability is a linear combination of a fixed number of terms and each of them has a limit as above.

Proof. Let $\tau$ be a subsequential limit of $\tau_{N}$. Denote by $Q=Q\left(A, n_{2}, \ldots, n_{k}\right)$ the event that $A \subset T$ (with the A's root corresponding to $T$ 's root) and that the part of $T$ in the $i^{\text {th }}$ external face of $A$ contains $n_{i}$ internal vertices. This is defined in the finite as well is the infinite setting (though we keep $n_{2}, \ldots, n_{k}<\infty$ ). In what follows $n_{1}$ denotes the number of vertices in the $1^{\text {st }}$ external face, i.e., $n_{1}=N-n-\sum_{i>1} n_{i}$. The probability of $Q$ is:

$$
\tau_{N}(Q)=\frac{\prod_{i=1}^{k} \varphi_{n_{i}, m_{i}}}{\varphi_{N-3,1}}
$$

and $\tau_{N}(A \subset T)$ is the sum over all possible vectors $\left(n_{i}\right)$ of this probability.

We first consider the limit:

$$
\lim _{N \rightarrow \infty} \tau_{N}\left(Q\left(A, n_{2}, \ldots, n_{k}\right)\right)=\left(\prod_{i>1} \varphi_{n_{i}, m_{i}}\right) \lim _{N \rightarrow \infty} \frac{\varphi_{n_{1}, m_{1}}}{\varphi_{N-3,1}}
$$




$$
\begin{aligned}
& =\left(\prod_{i>1} \varphi_{n_{i}, m_{i}}\right) \lim _{N \rightarrow \infty} \frac{C_{m_{1}} n_{1}^{-5 / 2} \alpha^{n_{1}}}{C_{1}(N-3)^{-5 / 2} \alpha^{N-3}} \\
& =\left(\prod_{i>1} \varphi_{n_{i}, m_{i}}\right) \frac{C_{m_{1}} \alpha^{-n-\sum_{i>1} n_{i}}}{C_{1} \alpha^{-3}} .
\end{aligned}
$$

Since the limit exists, it equals $\tau(Q)$. This may be written as:

$$
\tau(Q)=\frac{\alpha^{3-n}}{C_{1}} C_{m_{1}} \prod_{i>1} \varphi_{n_{i}, m_{i}} \alpha^{-n_{i}}
$$

Of course, a similar expression holds when the role of the 1 st face is filled by some other face, i.e., the sizes of all but the $i^{\text {th }}$ face are fixed.

Let $R_{i}=R_{i}(A)$ denote the event that $A \subset T$ and all the external faces of $A$ except possibly the $i^{\text {th }}$ one contain finitely many vertices. Obviously,

$$
R_{1}=\bigcup_{n_{2}, \ldots, n_{k}<\infty} Q\left(A, n_{2}, \ldots, n_{k}\right)
$$

Using (4.1) we get for any subsequential limit $\tau$ of $\tau_{N}$ :

$$
\tau\left(R_{1}\right)=\frac{\alpha^{3-n}}{C_{1}} C_{m_{1}} \prod_{i>1} Z_{m_{i}}
$$

and a similar formula for $R_{j}, j>1$. It is clear that $\tau\left(R_{i} \cap R_{j}\right)=0$ for $i \neq j$ in $\{1, \ldots, k\}$. Moreover, Corollary 3.4 (one end) implies $\tau\left(\{A \subset T\} \backslash \cup_{i} R_{i}\right)=0$. Hence,

$$
\tau(A \subset T)=\sum_{i=1}^{k} \tau\left(R_{i}\right)=\frac{\alpha^{3-n}}{C_{1}}\left(\prod Z_{m_{i}}\right) \sum \frac{C_{m_{i}}}{Z_{m_{i}}} .
$$

Proof of Theorem 1.7. Since for any $r$ the size of $B_{r}$ is tight in $N$ it suffices to show that for any possible ball $A$ the probability $\tau_{N}\left(B_{r}=A\right)$ converges to some limit as $N \rightarrow \infty$. However, we know that the ball $B_{r}$ satisfies the conditions of Proposition 4.10, and for any triangulation satisfying the conditions of Proposition 4.10 the limit exists.

Proof of Theorem 1.9. This follows from Theorem 1.7 and Corollary 3.4.

\section{Locality}

Next, we look at another basic property of the UIPT, namely locality. The meaning of locality is that isolated regions of the UIPT are almost independent. In the following, $R_{i}=R_{i}(A)$ will denote the event defined in the proof of Proposition 4.10.

Theorem 5.1. Let A be a finite rigid triangulation (for type III, with no edges between vertices on external faces). Assume A has $k$ external faces of sizes $m_{1}+2, \ldots, m_{k}+2$. Condition on the event $R_{i}(A)$, and let $T_{j}$ denote the component of the UIPT in the $j^{\text {th }}$ face. Then: 


\section{The triangulations $T_{j}$ are independent.}

2. $T_{i}$ has the same law as the UIPT of an $\left(m_{i}+2\right)$-gon (that is, the $N \rightarrow \infty$ limit of the uniform measure on rooted triangulations of an $\left(m_{i}+2\right)$-gon with $N$ internal vertices).

3. For $j \neq i, T_{j}$ has the same law as the free triangulation of an $\left(m_{j}+2\right)$-gon.

Proof. Without loss of generality, assume $i=1$. From Eqs. 4.2 and 4.1 we see that

$$
\tau\left(R_{1}\right)=\frac{\alpha^{3-n}}{C_{1}} C_{m_{1}} \prod_{j>1} Z_{m_{j}}
$$

and so

$$
\tau\left(\left|T_{j}\right|=n_{j} \text { for } j>1 \mid R_{1}\right)=\prod_{j>1} \frac{\varphi_{n_{j}, m_{j}} \alpha^{-n_{j}}}{Z_{m_{j}}} .
$$

Thus, we see that conditioned on $R_{1}(A)$ the sizes of the $T_{j}$ 's are independent, and $\left|T_{j}\right|$ is distributed like the free triangulation of an $\left(m_{j}+2\right)$-gon. Consider $\tau_{N}$. Conditioned on $Q\left(A, n_{2}, \ldots, n_{k}\right)$, since all possible triangulations of the sphere with the prescribed component sizes are equiprobable, the same holds for each component $T_{i}$. Thus, for any $N$ the joint distribution of $\left(T_{1}, \ldots, T_{j}\right)$ conditioned on their sizes is a product distribution. As $N \rightarrow \infty$, these joint distributions converge to the product distribution, where $T_{j}$ is uniform on triangulations with $\left|T_{j}\right|=n_{j}$.

Finally, the marginal of $T_{1}$ has size tending to infinity, and so converges to the UIPT of an $\left(m_{1}+2\right)$-gon.

\section{Ball Structure}

Recall that Theorem 5.1 tells us that conditioned on a sub-triangulation $T$, with some external faces, the probability that a face of size $m+2$ is the infinite one is proportional to $\frac{C_{m}}{Z_{m}}$. In the case of type II or III triangulations we have:

$$
\begin{gathered}
\frac{C_{m}^{2}}{Z_{m}^{2}}=\frac{(m+1)(m+2)(2 m+1)}{3 \sqrt{3 \pi}}, \\
\frac{C_{m}^{3}}{Z_{m}^{3}}=\frac{m(m+2)(2 m+1)}{6 \sqrt{6 \pi}},
\end{gathered}
$$

so in either case the probability of a face of size $m$ being the infinite face is roughly proportional to $\mathrm{m}^{3}$.

We wish to study the relation between the ball of radius $r$ and the ball of radius $r+1$. The ball of radius $r$ is a finite triangulation with any number of external faces with any combination of boundary sizes. Moving to $r+1$ we add in each outer face some triangles around its circumference. These added triangles can fill up the face, or they can split that face up into a number of sub-faces of different sizes. Figure 6.1a shows a ball with several finite faces, and the layer of the triangulation between radius $r$ and $r+1$ in the finite faces. The shaded areas are some of the faces of the ball of radius $r+1$. The infinite face may contain additional sub-faces. 

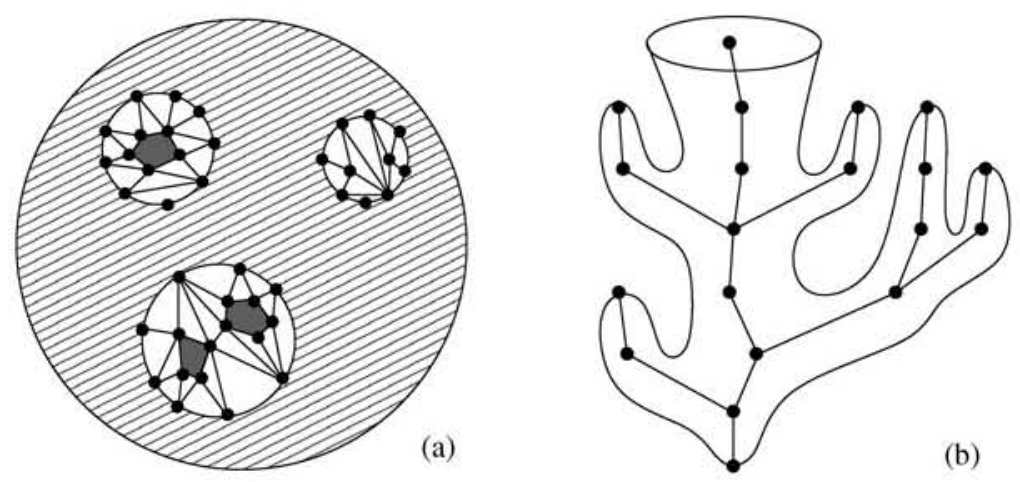

Fig. 6.1. (a) A possible ball in a planar triangulation. (b) A tree corresponding to a surface. Height corresponds to distance from the root

This gives rise to a tree-like structure for the triangulation, as in Fig. 6.1b. Each outer face of the ball of radius $r$ corresponds to a vertex in the $r^{\text {th }}$ level of the tree. The face corresponding to a child is contained in the face of the parent vertex. An infinite triangulation will yield an infinite tree. Similarly, if a triangulation is one ended, then so is the corresponding tree, i.e., the tree is composed of a single infinite branch from the root with finite sub-trees growing from it. Note that while any triangulation determines a tree, the converse is false. The tree does not determine the triangulation.

A vertex in the tree corresponds to an external face of some triangulation, so there are different types of vertices depending on the face sizes. Labeling each vertex with the boundary size of the corresponding face, we see that the UIPT gives rise to a multi-type tree process.

From Theorem 5.1 we see that if we condition the first $r$ levels of the tree and on which vertex in the $r^{\text {th }}$ level is in the infinite branch, then Theorem 5.1 tells us that the remaining sub-trees are independent. Thus, we see that at each level, one vertex, with a known distribution, has an infinite sub-tree above it, and the others have independent numbers of offspring of independent types. The tree process is thus just a multi-type Galton Watson process conditioned to survive. Without the conditioning we get the tree corresponding to a free triangulation of the sphere, which we know to be a.s. finite. However, since the free process has a power tail on its size, it is critical. Thus, the above description is just the construction of a critical Galton Watson process conditioned on survival (see [27]).

\section{Type Relations}

The two types of UIPT are part of a wider class of random planar objects satisfying common properties. This was first hinted at by the universality of the asymptotic formulas for counting various planar objects. Between type II and type III triangulations there is a more fundamental relation, enabling us to find a direct transformation between type II and type III triangulations. A similar transformation also holds between type I and type II triangulations as well as other pairs of classes of planar objects.

Roughly, the idea for passing from a type II triangulation to a type III triangulation is to take each double edge and to remove all the triangles and vertices inside it. The two edges are then glued together to get again a triangulation of the plane or sphere as the 
case may be. Conversely, to get from a type III triangulation to a type II one, we will take each edge and replace it with a double (or multiple) edge with some distribution on the triangulation inside the resulting 2-gons. Recall that we allowed the triangulation of the 2-gon with no internal vertices, and gluing it in a 2-gonal outer face meant gluing the two edges together. Thus, with some probability $\left(1 / Z_{0}=8 / 9\right.$ actually) this empty triangulation is used and the edge remains a single edge.

Both directions pose some difficulties. A 2-gon partitions a triangulation to two components. How do we decide which is the inside and which the outside? In an infinite triangulation of the plane we wish to contract the finite side, but for a finite triangulation of the sphere it is not so clear. Also, there is the possibility that the root of the triangulation is deleted in this way, and then a new root is needed.

In the opposite direction, there is the question of the distribution for the triangulation of the 2-gon added. The natural candidate in the infinite case is the free triangulation of the 2-gon. Again, in the finite setting things are more delicate. Since we define the infinite triangulation measures as limits of the finite ones, we need to find some transformation of the finite measures first.

For a type II triangulation $T$, when we contract 2-gons as described above, until no double edges remain, the result is a maximal (with respect to inclusion) 3-connected sub-triangulation, since the only way 2 vertices could separate the graph is by forming a 2-gon. Therefore, the transition can be summarized as taking a single maximal 3-connected subgraph of the triangulation. The natural choice for this is to take the 3 -connected component containing the root triangle. This also saves us the trouble of choosing a new root in the case that the old root was in one of the contracted 2-gons. Note that the root vertex or even the root edge is not enough, since 2 vertices may be in the intersection of two distinct 3 -connected components. However the 3 vertices of any triangle determine a unique 3-connected component.

Definition 7.1. Let $T$ be a rooted type II triangulation. Define $\tilde{T}$ to be the type III triangulation composed of the 3-connected component of the root in $T$, with all double edges identified into single edges. For a measure $v$ on rooted type II triangulations let $\tilde{v}$ be the resulting measure on type III triangulations, i.e., for any event $R$ :

$$
\tilde{v}(R)=v(\{T \mid \tilde{T} \in R\}) .
$$

This operation is known as taking the core of a structure [6]. In general, for two classes of rooted planar objects, one more restricted than the other, the core of a member of the wider class is its largest partial structure containing the root included in the smaller class (when it is unique).

Lemma 7.2. For any finite $N$, for some coefficients $a_{n, i}$ :

$$
\widetilde{\tau_{N}^{2}}=\sum_{i \leq N} a_{N, i} \tau_{i}^{3}
$$

In the limit, for some constants $a_{i}, a_{\infty}>0$ :

$$
\widetilde{\tau^{2}}=a_{\infty} \tau^{3}+\sum a_{i} \tau_{i}^{3}
$$

In the infinite case this means that the 3-connected component of the root is either a finite sphere triangulation with some distribution on the size where all triangulations of the same size are equiprobable, or it is an infinite triangulation. Conditioned on the latter case it is just the infinite type III UIPT. The asymptotics of the coefficients $a_{n, i}$ are described in [6]. 
Proof. Consider first the finite case. All we need to show is that any two type III triangulations have the same probability of appearing as the 3-connected component of the root in $\tau_{N}^{2}$, i.e., that for any triangulation $U$ the number of triangulations $T$ with $|T|=N$ and $\tilde{T}=U$ depends only on $|U|$.

This is clear, since any two triangulations of the same size have the same number of edges. Specifically, $U$ has $3|U|-6$ edges. Formally, if $Z_{0}^{2}(x)=\sum_{n} \varphi_{0, n}^{2} x^{n}$ is the generating function for triangulations of a 2 -gon, then the number of ways $U$ can come about is the coefficient of $x^{N-|U|}$ in $\left(Z_{0}(x)\right)^{3|U|-6}$, which is, of course, determined by $|U|$.

The infinite case follows from the finite case by taking a weak limit. The map $T \rightarrow \tilde{T}$ is continuous with respect to the topology on the spaces of type II and III triangulations. Since $\tau_{N}^{3}$ is supported on triangulations with $N$ vertices, they have disjoint supports for distinct $N$. Therefore, necessarily:

$$
\begin{aligned}
\widetilde{\tau^{2}} & =\lim _{N \rightarrow \infty} \widetilde{\tau_{N}^{2}} \\
& =\lim _{N \rightarrow \infty} \sum_{i} a_{N, i} \tau_{i}^{3} \\
& =\sum a_{i} \tau_{i}^{3}+a_{\infty} \tau^{3},
\end{aligned}
$$

where $a_{i}=\lim _{N} a_{N, i}$ must exist and

$$
a_{\infty}=\lim _{S \rightarrow \infty} \lim _{N \rightarrow \infty} \sum_{i>s} a_{N, i}
$$

is the part of the measure that tends to infinity. In the infinite case, we can also give an explicit formula for $a_{i}$. This is done in much the same way that we calculated the probability of a given ball when proving the limit of $\tau_{N}$ exists. Indeed, to find the probability $\widetilde{\tau^{2}}(T)$ for some type III triangulation $T$ with $|T|=n$ we just need to find the probability $\tau^{2}(T)$ when each edge is replaced by an external face of size 2. By Proposition 4.10 this is:

$$
(3 n-6) \alpha^{3-n}\left(Z_{0}^{2}\right)^{3 n-7} C_{0}^{2} / C_{1}^{2} .
$$

(A sphere triangulation with $n$ vertices has $3 n-6$ edges.) Substituting $Z_{0}^{2}=9 / 8$ and the values of $C$ this translates to:

$$
\frac{2^{19}}{3^{7}}(n-2)\left(\frac{27}{256}\right)^{n}
$$

Since there are $\varphi_{n-3,1}^{3}$ possible triangulations of size $n$, the probability $a_{n}=\tau^{2}(|\tilde{T}|=n)$ is:

$$
a_{n}=\frac{2^{20}(4 n-11) !}{3^{7}(n-3) !(3 n-7) !}\left(\frac{27}{256}\right)^{n} .
$$

In order to find $a_{\infty}$ we need to sum $a_{n}$. Since $(256 / 27)^{n} a_{n}$ is a linear combination of $\varphi_{n-3,1}$ and $n \varphi_{n-3,1}$, the generating function $A(t)=\sum a_{n}(256 t / 27)^{n}$ is a linear combination of $t^{-3} Z_{1}(t)$ and its derivative. Using that we find: $\sum a_{n}=1 / 2$ and the remainder:

$$
a_{\infty}=1 / 2
$$


Since we know that the UIPT is a.s. one ended, it has at most one infinite 3-connected component. The above calculations tell us more. We see that with probability $1 / 2$ the root triangle is part of the infinite 3-connected component. In fact, if the root is in a finite 3 -connected component, then this component has a number of 2-gonal external faces, and the infinite one contains a triangulation with the same LAW as the original UIPT. Iterating this we see that there is always a unique infinite 3-connected component, and with probability $1 / 2$ the root is part of it. This is an infinite version of an asymptotic result on finite triangulations found in [19]. In fact, we know now the distribution of the size of the 3-connected component of the root, as $a_{n}$ is the asymptotic probability that the component has size $n$.

How do we get back from the type III UIPT to the type II UIPT? We need to find the distribution of a UIPT conditioned on including an infinite triangulation. Theorem 5.1 deals with the UIPT conditioned on containing a finite sub-triangulation, and by conditioning on a growing subsequence of triangulations, we see that to get back from the infinite 3-connected component to the whole type II triangulation we need to replace each edge of $\tilde{T}$ with a free triangulation of a 2-gon.

Note that the expected number of triangles in a free triangulation of a 2-gon is twice the expected number of internal vertices and so is $2 / 3$ (again, this is the derivative of $Z_{0}^{3}(t)$ at $\left.\alpha^{-1}\right)$. Since a triangulation contains $3 / 2$ times as many edges as triangles, we see that in some sense in the resulting type II triangulation $1 / 2$ the triangles were in the original type III triangulation and $1 / 2$ were added.

As a consequence of this relation, some results on the type II UIPT are valid for type III as well. Those include the results on growth and on percolation derived in [4], among others.

Acknowledgement. We thank Itai Benjamini and Bálint Virág for inspiring conversations. Part of this research was done during visits of the first author to Microsoft Research. The first author thanks his hosts for these visits.

\section{References}

1. Ambjørn, J.: Quantization of Geometry. Lectures presented at the 1994 Les Houches Summer School "Fluctuating Geometries in Statistical Mechanics and Field Theory". arXiv : hep-th/9411179

2. Ambjørn, J., Durhuus, B., Jonsson, T.: Quantum Gravity, a Statitstical Field Theory Approach. Cambridge Monographs on Mathematical Physics, Cambridge: Cambridge University Press, 1997

3. Ambjørn, J., Watabiki, Y.: Scaling in quantum gravity. Nucl. Phys. B 445(1), 129-142 (1995)

4. Angel, O.: Growth and Percolation on the Uniform Infinite Planar Triangulation. In preparation

5. Banderier, C., Flajolet, P., Schaeffer, G., Soria, M.: Planar maps and Airy phenomena. In: Automata, lang. and prog. (Geneva, 2000), Lecture Notes in Comput. Sci., 1853, Berlin: Springer, 2000, pp. 388-402

6. Banderier, C., Flajolet, P., Schaeffer, G., Soria, M.: Random maps, coalescing saddles, singularity analysis, and Airy phenomena. In: Analysis of algorithms (Krynica Morska, 2000). Random Struc. Alg. 19(3-4), 194-246 (2001)

7. Bender, E.A., Compton, K.J., Richmond, B.L.: 0-1 laws for maps. Random Struc. Alg. 14(3), 215-237 (1999)

8. Bender, E.A., Richmond, B.L., Wormald, N.C.: Largest 4-connected components of 3-connected planar triangulations. Random Struc. Alg. 7(4), 273-285 (1995)

9. Benjamini, I., Schramm, O.: Recurrence of Distributional Limits of Finite Planar Graphs. Elec. J. Prob. 6, 23 (2001)

10. Boulatov, D.V., Kazakov, V.A.: The Ising Model on a Random Planar Lattice: the Structure of the Phase Transition and the Exact Critical Exponents. Phys. Lett. B 186(3-4), 379-384 (1987)

11. Bowick, M.J., Catterall, S.M., Thorleifsson, G.: Minimal Dynamical Triangulations of Random Surfaces. Phys. Lett. B 391(3-4), 305-309 (1997) 
12. Brown, W.G.: Enumeration of triangulations of the disk. Proc. London Math. Soc. (3) 14, 746-768 (1964)

13. Chassaing, P., Schaeffer, G.: Random Planar Lattices and Integrated Super-Brownian Excursion. arXiv:math.CO/0205226

14. David, F.: Simplicial Quantum Gravity and Random Lattices. In: Gravitation et quantifications (Les Houches, 1992), Amsterdam: North-Holland, Amsterdam, 1995, pp. 679-749, arXiv: hep-th/9303127

15. Di Francesco, P., Ginsparg, P., Zinn-Justin, J.: 2D Gravity and Random Matrices. Phys. Rep. 254(1-2), (1995)

16. Duplantier, B.: Random walks, polymers, percolation, and quantum gravity in two dimensions. In: STATPHYS 20 (Paris, 1998). Phys. A 263(1-4), 452-465 (1999)

17. Duplantier, B.: Random walks and quantum gravity in two dimensions. Phys. Rev. Lett. 81(25), 5489-5492 (1998)

18. Gao, Z., Richmond, B.L.: Root vertex valency distributions of rooted maps and rooted triangulations. Europ. J. Comb. 15(5), 483-490 (1994)

19. Gao, Z., Wormald, N.C.: The size of the largest component in random planar maps. Siam J. Disc. Math. 12(2), 217-228 (1999)

20. Goulden, I.P., Jackson, D.M.: Combinatorial enumeration. New York: Wiley, 1983

21. He, Z.X., Schramm, O.: Hyperbolic and parabolic packings. Disc. Comp. Geom. 14(2), 123-149 (1995)

22. Knizhnik, V.G., Polyakov, A.M., Zamolodchikov, A.B.: Fractal structure of 2D-quantum gravity. Mod. Phys. Lett. A 3(8), 819-826 (1988)

23. Koebe, P.: Kontaktprobleme der konformen abbildung, Berichte Verhande. Sächs. Akad. Wiss. Leipzig, Math. -Phys. Klasse 88, 141-164 (1936)

24. Lawler, G., Schramm, O., Werner, W.: Values of Brownian intersection exponents. I. Half-plane exponents. Acta Math. 187(2), 237-273 (2001)

25. Lawler, G., Schramm, O., Werner, W.: Values of Brownian intersection exponents. II. Plane exponents. Acta Math. 187(2), 275-308 (2001)

26. Lawler, G., Schramm, O., Werner, W.: Values of Brownian intersection exponents III: Two-sided exponents. Ann. Inst. H. Poincare Prob. Stat. 38(1), 109-123 (2002)

27. Lyons, R., Peres, Y.: Probability on Trees and Networks. www.math.gatech.edu/ rdlyons/prbtree/ prbtree.html

28. Richmond, B.L., Wormald, N.C.: Random triangulations of the plane. Euro. J. Comb. 9(1), 61-71 (1988)

29. Richmond, B.L., Wormald, N.C.: Almost all maps are asymmetric. J. Comb. Theory Ser. B 63(1), $1-7$ (1995)

30. Schaeffer, G.: Conjugaison s'arbres et cartes combinatoires aléatoires. PhD. Thesis, Université Bordeaux I, 1998, Bordeaux

31. Smirnov, S.: Critical percolation in the plane: conformal invariance, Cardy's formula, scaling limits. C. R. Acad. Sci. Paris Sć4. I Math. 333(3), 239-244 (2001)

32. Tutte, W.T.: A census of planar triangulations. Canad. J. Math. 14, 21-38 (1962)

33. Tutte, W.T.: A census of Hamiltonian polygons. Canad. J. Math. 14, 402-417 (1962)

34. Tutte, W.T.: A census of slicings. Canad. J. Math. 14, 708-722 (1962)

35. Tutte, W.T.: A census of planar maps. Canad. J. Math. 15, 249-271 (1963)

36. Tutte, W.T.: On the enumeration of convex polyhedra. J. Comb. The. Ser. B 28(2), 105-126 (1980)

Communicated by M. Aizenman 